\title{
Acknowledgement to Reviewers of $C$ in 2016
}

\author{
$C$ Editorial Office \\ MDPI AG, St. Alban-Anlage 66, 4052 Basel, Switzerland; c@mdpi.com \\ Published: 11 January 2017
}

The editors of $C$ would like to express their sincere gratitude to the following reviewers for assessing manuscripts in 2016.

We greatly appreciate the contribution of expert reviewers, which is crucial to the journal's editorial process. We aim to recognize reviewer contributions through several mechanisms, of which the annual publication of reviewer names is one. Reviewers receive a voucher entitling them to a discount on their next MDPI publication and can download a certificate of recognition directly from our submission system. Additionally, reviewers can sign up to the service Publons (https://publons.com) to receive recognition. Of course, in these intiatives we are careful not to compromise reviewer confidentiality. Many reviewers see their work as a voluntary and often unseen part of their role as researchers. We are grateful to the time reviewers donate to our journals and the contribution they make.

If you are interested in becoming a reviewer for $C$, see the link at the bottom of the webpage http://www.mdpi.com/reviewers.

The following reviewed for $C$ in 2016:

Adachi, Naoya
Akhavan, Omid
Alatalo, Sara-Maaria
Alexis, Frank
Andrieux, Fabrice
Barretta, Raffaele
Barroso-Bogeat, Adrián
Bello, Abdulhakeem
Brun, Nicolas
Casula, Maria F.
Cirillo, Giuseppe
Clyburne, Jason
Creamer, Anne Elise
Damodaran, Krishnan
David, Lamuel
Davies, Trevor
De La Escosura, Alfredo
Desvergne, Jean Pierre
Ding, Daowei
Dixon, David A.
Duong, Hai M.

\begin{tabular}{ll} 
Fischetti, Massimo V. & Manos, Manolis \\
Flox, Cristina & Mantripragada, Hari C. \\
Francia, Carlotta & Mokaya, Robert \\
Fu, Yongzhu & Nie, Zhenggang \\
Fujita, Shin-ichiro & Orbaek White, Alvin \\
Girousi, Stella & Park, Soo-Jin \\
Gough, Clair & Pozuelo, Javier \\
Guo, Chaohua & Qiang, Zhe \\
Hiete, Michael & Ramidi, Punnamchandar \\
Ikeda, Atsushi & Ratnakumar, B. V. \\
Iniesta, Jesus & Roger, Leblanc \\
Janegitz, Bruno Campos & Schubert, Tim \\
Jansen, Andrew N. & Sharifi, Tiva \\
Ji, Xiaobo & Simonin, Loic \\
Kalantar Zadeh, Kourosh & Singh, Gurpreet \\
Keeley, Gareth P. & Strano, Michael \\
Kickelbick, Guido & Strozzi, Matteo \\
Koskinen, Pekka & Takeguchi, Tatsuya \\
Lee, Cheng-Ming & Valentini, Luca \\
Lei, Weiwei & Vallés, Cristina \\
Locatelli, Clinio & Vidal, Hilario \\
\hline &
\end{tabular}


Videira, Romeu A.

Webley, Paul

Wilts, Henning
Xiong, Hui

Ye, Delai

Yeh, Yin-Ting
Zandi, Omid

Zhang, Minfang

Zhang, Sanliang

(c) 2017 by the authors; licensee MDPI, Basel, Switzerland. This article is an open access article distributed under the terms and conditions of the Creative Commons Attribution (CC-BY) license (http://creativecommons.org/licenses/by/4.0/). 